PROCEEDINGS OF THE

AMERICAN MATHEMATICAL SOCIETY

Volume 138, Number 4, April 2010, Pages 1425-1432

S 0002-9939(09)10169-7

Article electronically published on November 12, 2009

\title{
AN EXAMPLE OF AN ALMOST GREEDY BASIS IN $L^{1}(0,1)$
}

\author{
SMBAT GOGYAN
}

(Communicated by Michael T. Lacey)

\begin{abstract}
We give an explicit construction of an almost greedy basis of $L^{1}(0,1)$, complementing the results on existence of such a basis. The basis is described in terms of the Haar basis. We construct a quasi-greedy basis in a Banach space which is isomorphic to $L^{1}(0,1)$, and then we calculate an isomorphic image of a quasi-greedy basis.
\end{abstract}

\section{INTRODUCTION}

Let $\Psi=\left\{\psi_{k}\right\}_{k=1}^{+\infty}$ be a normalized basis in a Banach space $X$. For each $f \in X$ we have

$$
f=\sum_{k=1}^{\infty} c_{k}(f, \Psi) \psi_{k}
$$

where $\lim _{k \rightarrow \infty} c_{k}(f, \Psi)=0$. We define $\Lambda_{0}=\emptyset$; then for each $m \geq 1$ we inductively define sets $\Lambda_{m} \subset \mathbb{N}$ to satisfy

$$
\# \Lambda_{m}=m, \quad \Lambda_{m-1} \subset \Lambda_{m} \quad \text { and } \quad \min _{k \in \Lambda_{m}}\left|c_{k}(f, \Psi)\right| \geq \max _{k \notin \Lambda_{m}}\left|c_{k}(f, \Psi)\right| .
$$

Note that the sets $\Lambda_{m}$ are not uniquely determined. Denote the set of all such sequences $\left\{\Lambda_{m}\right\}$ by $D(f)$. For any $\Lambda \in D(f)$ we put

$$
G_{m}(f)=G_{m}(f, \Psi)=G_{m}(f, \Psi, \Lambda)=\sum_{k \in \Lambda_{m}} c_{k}(f, \Psi) \psi_{k} .
$$

This nonlinear method of approximation is known as a Thresholding Greedy Algorithm (TGA) or as a Greedy algorithm (see for example [6]).

In fact $G_{m}(f)$ can be realized by the following procedure: take the expansion (11) and form a sum of $m$ terms with the biggest $\left|c_{k}(f, \Psi)\right|$. If the basis $\Psi$ is unconditional, then obviously

$$
\left\|G_{m}(f, \Psi, \Lambda)\right\|_{X} \leq C \cdot\|f\|_{X}
$$

with a constant $C$ independent of $f, m$ and $\Lambda$.

Definition. A basis $\Psi=\left\{\psi_{k}\right\}_{k=1}^{+\infty}$ is called quasi-greedy for $X$ if there exists a constant $C$ such that for any $f \in X$ and for any $\Lambda \in D(f)$ the inequality (2) holds for any $m \in \mathbb{N}$.

The following theorem was proved in [8].

Received by the editors April 19, 2009, and, in revised form, August 14, 2009.

2000 Mathematics Subject Classification. Primary 42C30.

Key words and phrases. Haar system, greedy algorithm, quasi-greedy, almost greedy, $L^{1}(0,1)$.

(C)2009 American Mathematical Society Reverts to public domain 28 years from publication 
Theorem A. A basis $\Psi=\left\{\psi_{k}\right\}_{k=1}^{+\infty}$ is quasi-greedy for $X$ if and only if for any $f \in X$ there exists $\Lambda \in D(f)$ such that

$$
\lim _{m \rightarrow+\infty}\left\|f-G_{m}(f, \Psi, \Lambda)\right\|_{X}=0 .
$$

Definition 2. A basis $\Psi=\left\{\psi_{k}\right\}_{k=1}^{+\infty}$ is called almost greedy for $X$ if there exists a constant $C$ such that for all $f \in X$ and $m \in \mathbb{N}$ we have

$$
\left\|f-G_{m}(f, \Psi)\right\|_{X} \leq C \inf _{A \subset \mathbb{N},|A|=m}\left\{\left\|f-\sum_{j \in A} c_{j}(f, \Psi) \psi_{j}\right\|\right\} .
$$

It follows from Definition 2 that every almost greedy basis is also a quasi-greedy basis. It was proved in [2] that $\Psi$ is almost greedy if and only if $\Psi$ is quasi-greedy and democratic. Recall that $\Psi$ is democratic if for some $C \geq 1$ we have

$$
\left\|\sum_{j \in A} \psi_{j}\right\| \leq C\left\|\sum_{j \in B} \psi_{j}\right\| \text { whenever } \quad|A| \leq|B| .
$$

Definition 3. A basis $\Psi=\left\{\psi_{k}\right\}_{k=1}^{+\infty}$ is called greedy for $X$ if there exists a constant $C$ such that for all $f \in X$ and $m \in \mathbb{N}$ we have

$$
\left\|f-G_{m}(f, \Psi)\right\|_{X} \leq C \inf \left\{\left\|f-\sum_{j \in A} a_{j} \psi_{j}\right\| \quad: \quad a_{j} \in \mathbb{R},|A|=m\right\} .
$$

It was proved in 6 that a basis is greedy if and only if it is unconditional and democratic. So we have the following relations:

$$
\begin{aligned}
& \text { Greedy } \Longrightarrow \text { Unconditional } \Longrightarrow \text { Quasi-greedy, } \\
& \text { Greedy } \Longrightarrow \text { Almost greedy } \Longrightarrow \text { Quasi-greedy, } \\
& \text { Greedy }=\text { Unconditional }+ \text { Democratic }, \\
& \text { Almost greedy }=\text { Quasi-greedy }+ \text { Democratic. }
\end{aligned}
$$

It was proved by V. N. Temlyakov (see [7]) that the Haar system is a greedy basis in $L^{p}(0,1), 1<p<\infty$.

It is known that there is no unconditional basis in $L^{1}(0,1)$. But it was proved in [1] that there is an almost greedy basis in $L^{1}(0,1)$. That proof was not constructive, and that basis was not constructed. In [3] it was shown that the Haar system is not a quasi-greedy basis in $L^{1}(0,1)$. The same result for general Haar systems and for the Franklin system was proved in [4] and [5] correspondingly.

In this paper we give an example of an almost greedy basis in $L^{1}(0,1)$. We denote

$$
M_{i}=2^{i}-i-\sum_{p: 2^{p}+1<i}\left(2^{2^{p}+1}-1\right), i=0,1, \ldots
$$

It is easy to check that

$$
M_{0}=1 \text { and } M_{i+1}-M_{i}= \begin{cases}0, & \text { if } i=2^{p}+1 \text { for some natural } p, \\ 2^{i}-1, & \text { otherwise. }\end{cases}
$$

Let $\left\{h_{n}\right\}$ be a classical Haar system normalized in $L^{1}(0,1)$ and let $\psi_{i}=h_{2^{i}+2}$ (i.e. the Haar function with support $\left[2^{-i}, 2^{1-i}\right.$ ). 
Let $i \geq 0$ and $i \neq 2^{p}+1$ for any $p \geq 0$. For any $0 \leq k \leq M_{i+1}-M_{i}=2^{i}-1$ we define

$$
f_{(i, k)}= \begin{cases}h_{i+1}-\frac{1}{2^{i}} \sum_{j=0}^{2^{i}-2} \psi_{M_{i}+j}+\psi_{M_{i}+k}, & \text { if } k<2^{i}-1, \\ h_{i+1}-\frac{1}{2^{i}} \sum_{j=0}^{2^{i}-2} \psi_{M_{i}+j}, & \text { if } k=2^{i}-1 .\end{cases}
$$

Theorem 1. The system $\left\{f_{(i, k)}\right\}$ is an almost greedy basis in $L^{1}(0,1)$.

We construct a quasi-greedy basis in an auxiliary Banach space and show that it is an isomorphic image of $\left\{f_{(i, k)}\right\}$.

\section{Preliminaries and AUXiliary Results}

We need results from [1. Let $X$ be a Banach space with a basis $\left\{b_{n}\right\}$. By passing to an equivalent norm we may assume that $\left\{b_{n}\right\}$ is normalized and bimonotone. Let $S$ be a 1 -symmetric and 1-unconditional sequence space with basis $\left\{e_{i}\right\}, e_{i}=\left\{\delta_{i j}\right\}$. Let $\left\{e_{i}^{*}\right\}$ be the sequence of biorthogonal functionals in $S^{*}$. Define

$$
f(n)=\left\|e_{1}+\ldots+e_{n}\right\|_{S}
$$

and

$$
g(n)=\frac{n}{f(n)}=\left\|e_{1}^{*}+\ldots+e_{n}^{*}\right\|_{S^{*}}
$$

We assume that $\left\{e_{i}\right\}$ is not equivalent to the unit vector basis of $c_{0}$. Thus,

$$
f(n) \rightarrow \infty \quad \text { as } n \rightarrow \infty .
$$

For $n \geq 1$, let $\sigma_{n}=\left[2^{n-1} ; 2^{n}-1\right]$,

$$
v_{n}=\frac{1}{f\left(2^{n-1}\right)} \sum_{k \in \sigma_{n}} e_{k} \quad \text { and } \quad v_{n}^{*}=\frac{1}{g\left(2^{n-1}\right)} \sum_{k \in \sigma_{n}} e_{k}^{*} .
$$

Let $P$ be the norm-one projection on $S$ defined by

$$
P \xi=\sum_{n=1}^{\infty}\left\langle\xi, v_{n}^{*}\right\rangle v_{n}
$$

and let $Q=I-P$. Define a norm on $c_{00}$ by

$$
\|\xi\|_{Y}=\left\|\sum_{n=1}^{\infty}\left\langle\xi, v_{n}^{*}\right\rangle b_{n}\right\|_{X}+\|Q \xi\|_{S}
$$

and then complete it to obtain a sequence space $Y$.

The following statements were proved in [1].

Statement 1 (Proposition 6.1). Suppose that $\left\{b_{n}\right\}$ is a bimonotone basis for $X$. Then $\left\{e_{n}\right\}$ is a basis for $Y$ such that

$$
\frac{1}{8} \sup _{n} \eta_{n} f(n) \leq\|\xi\|_{Y} \leq 6 \sum_{n=1}^{\infty} \frac{f(n)}{n} \eta_{n}
$$

for all real scalars $\xi=\left(\xi_{n}\right)$ in $c_{00}$, where $\left\{\eta_{i}\right\}$ is the nonincreasing rearrangement of $\left\{\left|\xi_{i}\right|\right\}$.

Statement 2. Suppose that $X$ is a Banach space with a basis that contains a complemented subspace isomorphic to $S$. Then $Y \sim X$. 
Statement 3 (Theorem 7.1). Suppose that $\left\{b_{n}\right\}$ is a basis for $X$ and $S=\ell_{1}$. Then $\left\{e_{n}\right\}$ is a quasi-greedy basis for $Y$.

Let us apply Statements 1-3 when $X=L^{1}(0,1)$ and $S=\ell_{1},\left\{b_{i}\right\}=\left\{h_{i}\right\}$ (the Haar system).

Lemma 1. The system $\left\{e_{i}\right\}$ is an almost greedy basis in $Y$ and

$$
Q\left(e_{2^{i}+k}\right)=(\underbrace{0, \ldots, 0}_{2^{i}-1}, \underbrace{-2^{-i}, \ldots,-2^{-i}}_{k}, 1-2^{-i}, \underbrace{-2^{-i}, \ldots,-2^{-i}}_{2^{i}-k-1}, 0,0 \ldots)
$$

for all $0 \leq k<2^{i}$.

Proof. We have $f(n)=n$ and $g(n)=1$. Let $\xi=\sum_{k \in A} e_{k}$ with $|A|=m$. Then

$$
\eta_{n}= \begin{cases}1, & n \leq m \\ 0, & n>m\end{cases}
$$

Hence, according to Statement 1 we conclude that

$$
\frac{m}{8} \leq\left\|\sum_{k \in A} e_{k}\right\|_{Y} \leq 6 m .
$$

Hence $\left\|\sum_{k \in A} e_{k}\right\|_{Y} \asymp|A|$. So $\left\{e_{n}\right\}$ is democratic in $Y$. Combined with Statement 3 we conclude that $\left\{e_{n}\right\}$ is an almost greedy basis. Equality (4) immediately follows from the definition of $Q$. Lemma 1 is proved.

According to the definition of $Y$ there exists an isomorphic operator R : $Y \mapsto$ $L^{1}(0,1) \oplus Q\left(\ell_{1}\right)$ such that

$$
\mathrm{R}\left(e_{2^{i}+k}\right)=\left(h_{i+1}, Q\left(e_{2^{i}+k}\right)\right)
$$

for every $k=0, \ldots, 2^{i}-1 ; i=0,1, \ldots$

\section{ISOMORPHIC OPERATORS}

Let $\left\{M_{i}\right\}$ be the sequence defined by (3) and let $\Lambda_{n}=\left[2^{n-1}, 2^{n}-2\right]$. Denote

$$
\Lambda=\left\{n_{1}, n_{2}, n_{3}, \ldots\right\}=\bigcup_{p=0}^{\infty} \Lambda_{2^{p}+2} \text { and }\left\{m_{1}, m_{2}, \ldots\right\}=\left(\bigcup_{n=1}^{\infty} \Lambda_{n}\right) \backslash \Lambda
$$

where sequences $\left\{n_{i}\right\}$ and $\left\{m_{i}\right\}$ are increasing. For every $\left\{a_{i}\right\} \in Q\left(\ell_{1}\right)$ we define the operator $\mathrm{S}: Q\left(\ell_{1}\right) \mapsto \ell_{1} \oplus \ell_{1}$ by

$$
\mathrm{S}\left(\left\{a_{i}\right\}\right)=\left(\left\{a_{m_{1}}, a_{m_{2}}, \ldots\right\},\left\{a_{n_{1}}, a_{n_{2}}, \ldots\right\}\right) .
$$

Since $\left\{a_{i}\right\} \in Q\left(\ell_{1}\right)$ if and only if $\left\{a_{i}\right\} \in \ell_{1}$ and $a_{2^{n}-1}=-\sum_{i \in \Lambda_{n}} a_{i}$ for all natural $n$, we conclude that $\mathrm{S}$ is an isomorphic operator. The next lemma follows from the definitions of $Q$ and S. 
Lemma 2. 1) Let $i \neq 2^{p}+1$ for any $p \geq 0$. Then

$$
\begin{aligned}
& \mathrm{S}\left(Q\left(e_{2^{i}+k}\right)\right) \\
& = \begin{cases}\left((\underbrace{0, \ldots, 0}_{M_{i}-1}, \underbrace{-2^{-i}, \ldots,-2^{-i}}_{k}, 1-2^{-i}, \underbrace{-2^{-i}, \ldots,-2^{-i}}_{2^{i}-k-2}, 0 \ldots),\{0\}\right), & \text { if } k<2^{i}-1, \\
\left((\underbrace{0, \ldots, 0}_{M_{i}-1}, \underbrace{-2^{-i}, \ldots,-2^{-i}}_{2^{i}-1}, 0 \ldots),\{0\}\right), & \text { if } k=2^{i}-1 .\end{cases} \\
& \text { 2) Let } i=2^{p}+1 \text { for some } p \geq 0 \text { and } 0 \leq k<2^{i} . \text { Then } \\
& \mathrm{S}\left(Q\left(e_{2^{i}+k}\right)\right) \\
& = \begin{cases}\left(\{0\},(\underbrace{0, \ldots, 0}_{2^{i}-i-M_{i}}, \underbrace{-2^{-i}, \ldots,-2^{-i}}_{k}, 1-2^{-i}, \underbrace{-2^{-i}, \ldots,-2^{-i}}_{2^{i}-k-2}, 0 \ldots)\right), & \text { if } k<2^{i}-1, \\
\left(\{0\},(\underbrace{0, \ldots, 0}_{2^{i}-i-M_{i}}, \underbrace{-2^{-i}, \ldots,-2^{-i}}_{2^{i}-1}, 0,0 \ldots)\right), & \text { if } k=2^{i}-1 .\end{cases}
\end{aligned}
$$

Functions $\psi_{i}$ have disjoint supports $\left(\operatorname{supp}\left(\psi_{i}\right)=\left(2^{-i}, 2^{1-i}\right)\right)$. Therefore we have

$$
\left\|\sum_{i=1}^{\infty} a_{i} \psi_{i}\right\|_{L^{1}(0,1)}=\left\|\left\{a_{i}\right\}\right\|_{\ell_{1}} .
$$

Denote

$$
Z=\left\{f \in L^{1}(0,1) \quad: \quad \int_{0}^{1} f(t) \psi_{n}(t) d t=0 \text { for all } n \in \mathbb{N}\right\} .
$$

Let us define the operator $\mathrm{T}: L^{1}(0,1) \mapsto\left(Z, \ell_{1}\right)$ by the formula

$$
\mathrm{T}(f)=\mathrm{T}\left(\sum_{n=1}^{\infty} a_{n} h_{n}\right)=\left(\sum_{n=1, n \neq 2^{i}+2}^{\infty} a_{n} h_{n},\left\{a_{2^{i}+2}\right\}\right) .
$$

Note that

$$
\mathrm{T}\left(h_{n}\right)= \begin{cases}\left(0,\left\{\delta_{p+1, j}\right\}\right), & \text { if } n=2^{p}+2, \\ \left(h_{n},\{0\}\right), & \text { otherwise }\end{cases}
$$

The next lemma is obvious.

Lemma 3. The operator $\mathrm{T}$ represents an isomorphism between $L^{1}(0,1)$ and $Z \oplus \ell_{1}$.

4. An ALmost GREedy Basis In $L^{1}(0,1)$

In the proof of Statement 2 the following isomorphic chain was used:

$$
\begin{aligned}
Y \sim X \oplus Q(S) & \sim Z \oplus S \oplus Q(S) \\
& \sim Z \oplus S \oplus P(S) \oplus Q(S) \\
& \sim Z \oplus S \oplus S \sim Z \oplus S \sim X .
\end{aligned}
$$


In the proof of Theorem 1 we will use the following:

$$
\begin{array}{rlrl}
Y & \sim L^{1}(0,1) \oplus Q\left(\ell_{1}\right) & \\
& \sim Z \oplus \ell_{1} \oplus Q\left(\ell_{1}\right) & & (\text { by operator } \mathrm{T}) \\
& \sim Z \oplus \ell_{1} \oplus \ell_{1} \oplus \ell_{1} & & (\text { by operator } \mathrm{S}) \\
& \sim L^{1}(0,1) \oplus \ell_{1} \oplus \ell_{1} . & & (\text { by operator } \mathrm{T}) .
\end{array}
$$

Proof of Theorem 1. For $y \in Y$ denote

$$
\begin{aligned}
& \mathrm{R}(y)=\left(y_{1}, q_{1}\right), \text { where } y_{1} \in L^{1}(0,1), q_{1} \in Q\left(\ell_{1}\right) ; \\
& \mathrm{T}\left(y_{1}\right)=\left(z_{1}, a_{1}\right), \text { where } z_{1} \in Z, a_{1} \in \ell_{1} ; \\
& \mathrm{S}\left(q_{1}\right)=\left(a_{2}, a_{3}\right), \text { where } a_{2}, a_{3} \in \ell_{1} ; \\
& \mathrm{T}^{-1}\left(z_{1}, a_{2}\right)=y_{2}, \text { where } y_{2} \in L^{1}(0,1) ;
\end{aligned}
$$

and set

$$
\mathrm{D}(y)=\left\{y_{2}, a_{1}, a_{3}\right\}
$$

It is clear that $\mathrm{D}$ is an isomorphic operator from $Y$ to $L^{1}(0,1) \oplus \ell_{1} \oplus \ell_{1}$. Now, let us calculate $\mathrm{D}\left(e_{n}\right)$. Let $n=2^{i}+k$ with $0 \leq k<2^{i}$. According to (5) we have

$$
\mathrm{R}\left(e_{2^{i}+k}\right)=\left(h_{i+1}, Q\left(e_{2^{i}+k}\right)\right),
$$

which means that $y_{1}=h_{i+1}$ and $q_{1}=Q\left(e_{2^{i}+k}\right)$. We consider four cases.

Case 1. $i=2^{p}+1$ for some $p \geq 0$ and $k<2^{i}-1$. According to (7)

$$
\begin{aligned}
\left(z_{1}, a_{1}\right) & =\mathrm{T}\left(h_{i+1}\right)=\left(0,\left\{\delta_{p+1, j}\right\}\right), \\
\left(a_{2}, a_{3}\right) & =\mathrm{S}\left(Q\left(e_{2^{i}+k}\right)\right) \\
& =\left(\{0\},\{\underbrace{0, \ldots, 0}_{2^{i}-i-M_{i}}, \underbrace{-2^{-i}, \ldots,-2^{-i}}_{k}, 1-2^{-i}, \underbrace{-2^{-i}, \ldots,-2^{-i}}_{2^{i}-k-2}, 0 \ldots\}\right), \\
y_{2} & =\mathrm{T}^{-1}\left(z_{1}, a_{2}\right)=\mathrm{T}^{-1}(0,\{0\})=0 .
\end{aligned}
$$

Hence

$$
\mathrm{D}\left(e_{2^{i}+k}\right)=\left(0,\left\{\delta_{p+1, j}\right\},\{\underbrace{0, \ldots, 0}_{2^{i}-i-M_{i}}, \underbrace{-2^{-i}, \ldots,-2^{-i}}_{k}, 1-2^{-i}, \underbrace{-2^{-i}, \ldots,-2^{-i}}_{2^{i}-k-2}, 0 \ldots\}\right) .
$$

Case 2. $i=2^{p}+1, k=2^{i}-1$.

$$
\begin{aligned}
\left(z_{1}, a_{1}\right) & =\mathrm{T}\left(h_{i+1}\right)=\left(0,\left\{\delta_{p+1, j}\right\}\right), \\
\left(a_{2}, a_{3}\right) & =\mathrm{S}\left(Q\left(e_{2^{i}+k}\right)\right)=\left(\{0\},\{\underbrace{0, \ldots, 0}_{2^{i}-i-M_{i}}, \underbrace{-2^{-i}, \ldots,-2^{-i}}_{2^{i}-1}, 0 \ldots\}\right), \\
y_{2} & =\mathrm{T}^{-1}\left(z_{1}, a_{2}\right)=\mathrm{T}^{-1}(0,\{0\})=0 .
\end{aligned}
$$

Hence $\mathrm{D}\left(e_{2^{i}+k}\right)=\left(0,\left\{\delta_{p+1, j}\right\},\{\underbrace{0, \ldots, 0}_{2^{i}-i-M_{i}}, \underbrace{-2^{-i}, \ldots,-2^{-i}}_{2^{i}-1}, 0 \ldots\}\right)$. 
Case $3 . i \neq 2^{p}+1, k \neq 2^{i}-1$.

$$
\begin{aligned}
\left(z_{1}, a_{1}\right) & =\mathrm{T}\left(h_{i+1}\right)=\left(h_{i+1},\{0\}\right), \\
\left(a_{2}, a_{3}\right) & =\mathrm{S}\left(Q\left(e_{2^{i}+k}\right)\right) \\
& =\left((\underbrace{0, \ldots, 0}_{M_{i}-1}, \underbrace{-2^{-i}, \ldots,-2^{-i}}_{k}, 1-2^{-i}, \underbrace{-2^{-i}, \ldots,-2^{-i}}_{2^{i}-k-2}, 0 \ldots),\{0\}\right), \\
y_{2} & =\mathrm{T}^{-1}\left(z_{1}, a_{2}\right) \\
& =\mathrm{T}^{-1}\left(h_{i+1},(\underbrace{0, \ldots, 0}_{M_{i}-1}, \underbrace{-2^{-i}, \ldots,-2^{-i}}_{k}, 1-2^{-i}, \underbrace{-2^{-i}, \ldots,-2^{-i}}_{2^{i}-k-2}, 0 \ldots)\right) \\
& =h_{i+1}-\frac{1}{2^{i}} \sum_{j=M_{i}}^{M_{i}+2^{i}-2} \psi_{j}+\psi_{M_{i}+k}=h_{i+1}-\frac{1}{2^{i}} \sum_{j=M_{i}}^{M_{i+1}-1} \psi_{j}+\psi_{M_{i}+k} .
\end{aligned}
$$

Therefore $\mathrm{D}\left(e_{2^{i}+k}\right)=\left(f_{(i, k)},\{0\},\{0\}\right)$.

Case 4. $i \neq 2^{p}+1, k=2^{i}-1$.

$$
\begin{aligned}
\left(z_{1}, a_{1}\right) & =\mathrm{T}\left(h_{i+1}\right)=\left(h_{i+1},\{0\}\right), \\
\left(a_{2}, a_{3}\right) & =\mathrm{S}\left(Q\left(e_{2^{i}+k}\right)\right)=\left((\underbrace{0, \ldots, 0}_{M_{i}-1}, \underbrace{-2^{-i}, \ldots,-2^{-i}}_{2^{i}-1}, 0 \ldots),\{0\}\right), \\
y_{2} & =\mathrm{T}^{-1}\left(z_{1}, a_{2}\right)=\mathrm{T}^{-1}\left(h_{i+1},(\underbrace{0, \ldots, 0}_{M_{i}-1}, \underbrace{-2^{-i}, \ldots,-2^{-i}}_{2^{i}-1}, 0 \ldots)\right) \\
& =h_{i+1}-\frac{1}{2^{i}} \sum_{j=M_{i}}^{M_{i+1}-1} \psi_{j} .
\end{aligned}
$$

Therefore $\mathrm{D}\left(e_{2^{i}+k}\right)=\left(f_{(i, k)},\{0\},\{0\}\right)$.

Finally we have

$$
\mathrm{D}\left(e_{2^{i}+k}\right)= \begin{cases}(0,\{\ldots\},\{\ldots\}) & , \text { if } i=2^{p}+1, \\ \left(f_{(i, k)},\{0\},\{0\}\right) & , \text { if } i \neq 2^{p}+1 .\end{cases}
$$

Since $\left\{\mathrm{D}\left(e_{2^{i}+k}\right)\right\}$ is an almost-greedy basis in $L^{1}(0,1) \oplus \ell_{1} \oplus \ell_{1}$, then the system $\left\{f_{(i, k)}\right\}$ is an almost greedy basis in $L^{1}(0,1)$. Theorem 1 is proved.

\section{ACKNOWLEDGMENT}

The author is thankful to Michael Lacey for his suggestions and for help in the preparation of this paper. 


\section{REFERENCES}

[1] S. J. Dilworth, N. J. Kalton, D. Kutzarova, On the existence of almost greedy bases in Banach spaces, Studia Mathematics 159(2003), N1, 67-101. MR2030904 (2005a:46021)

[2] S.J. Dilworth, N.J. Kalton, D. Kutzarova, V.N. Temlyakov, The thresholding greedy algorithm, greedy bases, and duality, Constr. Approx. 19(2003), N4, 575-597. MR1998906 (2004e:41045)

[3] S.J. Dilworth, D. Kutzarova, P. Wojtaszczyk, On approximate $l_{1}$ systems in Banach spaces, J. Approx. Theory 114(2002), N2, 214-241. MR.1883407(2002k:46024)

[4] S. Gogyan, Greedy algorithm with regard to Haar subsystems, East J. Approx. 11(2005), N2, 221-236. MR.2151616 (2006c:41041)

[5] G. G. Gevorkyan, A. Kamont, Two remarks on quasi-greedy bases in the space $L_{1}$, J. Contemp. Math. Anal. 40(2005), N1, 2-14. MR.2265671 (2007k:46049)

[6] S.V. Konyagin, V.N. Temlyakov, A remark on greedy approximation in Banach spaces, East J. Approx. 5(1999), 365-379. MR1716087 (2000j:46020)

[7] V. N. Temlyakov, The best m-term approximation and greedy algorithms, Advances in Comp. Math. 8(1998), N3, 249-265. MR.1628182 (99f:41037)

[8] P. Wojtaszczyk, Greedy algorithms for general biorthogonal systems, J. Approx. Theory 107(2000), N2, 293-314. MR.1806955 (2001k:46017)

Institute of Mathematics, National Academy of Sciences, 24B Marshal Baghramian Avenue, 0019 Yerevan, Armenia 